Laryngoscopy in the Setting of the Predicted Difficult Airway", Anesthesiology; 116: 515-7.

8. Roya Ymul MD et al (2016), "Comparison of three video laryngoscopy devices to direct laryngoscopy for intubating obese patients: a randomized trial", Journal of Clinical Anesthesia
31, 71-77.

9. Roya Yumul MD, Ofelia L. Elvir MD, Paul F.White PhD et al (2016), "Comparison of the C-MAC video laryngoscope to a flexible fiberoptic scope for intubation with cervical spine immobilization", Journal of Anesthesia 31, 46-52.

\title{
Độ CỨNG VICKERS VÀ CẤU TRÚC BỀ MẶT SỨ LITHIUM DISILICATE TRƯỚC VÀ SAU XỬ LÝ AXIT HYDROFLUORIC
}

\section{TÓM TẮT}

Mục tiêu: Nghiên cứu đánh giá độ cứng Vickers và cấu trúc bề mặt của sứ thuỷ tinh lithium disilicate trước và sau khi xử lý với axit hydrofluoric (HF) $5 \%$. Đối tượng và phương pháp nghiên cứu: 52 đĩa tròn sứ thuy tinh lithium disilicate (26 đĩa sứ GC Initial Lisi Press-LP và 26 đĩa sứ IPS e.max Press-EP) có kích thước $4 \times 2 \mathrm{~mm}$ được chuẩn bị bằng kỹ thuât ép nóng. Sau khi đánh bóng bề mặt, một nửa số đĩa sứ được xử lý bề mă̆t với HF 5\%. Sau đó, 40 đĩa sứ được dùng để đánh giá đô cứng Vickers và 12 đĩa dùng để quan sát bề mặt dưới kính hiển vi điện tử quét. Kết quả: Sau khi xử lý bề mătt với HF 5\%, độ cứng Vickers của 2 nhóm nghiên cứu đều giảm có ý nghĩa thống kê và nhóm LP có độ cứng Vickers lớn hơn có ý nghĩa thống kê so với nhóm EP. Hình ảnh SEM cho thây cấu trúc bề mă̆t của nhóm LP có các tinh thể với kích thước nhỏ, đều hơn so với nhóm EP. Kết luận: Việc xử lý với $\mathrm{HF} 5 \%$ làm giảm độ cứng bề mặt của sứ lithium disilicate và độ cứng của LP lớn hơn EP.

Tư khoá: Độ cứng Vickers, kính hiển vi điện tử quét, sứ lithium disilicate, axit hydrofluoric

\section{SUMMARY \\ THE VICKERS HARDNESS AND SURFACE STRUCTURE OF LITHIUM DISILICATE CERAMIC BEFORE AND AFTER HYDROFLUORIC ACID TREATMENT}

Objective: The study was to evaluate the Vickers Hardness and the surface structure of lithium disilicate glass ceramics before and after treatment with $5 \%$ hydrofluoric acid (HF). Methods: Fifty-two round lithium disilicate ceramic discs of $4 \times 2 \mathrm{~mm}$ (26 discs of GC Initial Lisi Press-LP and 26 discs of IPS E.max Press-EP) was prepared by heat-pressing technique. Half of each group then were etched with $5 \% \mathrm{HF}$ in 20 seconds after surface polishing. Then, 40 ceramic discs were used for Vickers Hardness evaluation and 12 ceramic discs were used to observe the surface structure using scanning electron microscope (SEM).

*Đại học Y Dược Tp. Hồ Chí Minh

Chịu trách nhiệm chính: Trân Xuân Vĩnh

Email: vinhdentist@yahoo.com

Ngày nhận bài: 7.5.2021

Ngày phản biện khoa học: 24.6.2021

Ngày duyệt bài: 7.7.2021

\section{Trần Lê Khoa*, Trần Xuân Vĩnh*}

Results: After surface treatment with $5 \% \mathrm{HF}$, the Vickers Hardness of the two groups decreased significantly and Vickers Hardness of LP group was significantly higher than that of EP group. SEM observation showed the surface of LP group have smaller sized lithium disilicate crystals and more uniform than that of EP group. Conclusion: The surface treatment with 5\% HF decreased the Vickers Hardness of lithium disilicate ceramics and the surface hardness of LP was higher than that of EP.

Keywords: Vickers Hardness, scanning electron microscope, hydrofluoric acid, lithium disilicate ceramic.

\section{I. ĐẶT VẤN ĐỀ}

Nhu cầu thẩm mỹ trong nha khoa ngày càng tăng kèm theo những lo ngại gia tăng liên quan đến dị ứng kim loại [1]. Việc sử dụng phục hồi không chứa kim loại được kỳ vọng sẽ tái tạo màu sắc, độ bóng, độ trong tự nhiên của răng thật. Phục hình toàn sứ được sử dụng rộng rãi cho mão răng, cầu răng, inlay, onlay và mặt dán ở cả vùng răng trước và sau nhờ đặc tính cơ học tốt và tính thẩm mỹ cao.

Các phương pháp chễ tác sứ như sứ thiêu kết, ép nóng và công nghệ thiết kế/sản xuất hỗ trợ máy tính (CAD/CAM) được áp dụng để xử lý các vật liệu sứ khác nhau. Sự phát triển không ngừng của công nghệ vật liệu sứ dẫn đến việc tạo ra các phục hình giống với răng thật, đạt được kết quả lâu dài. Lithium disilicate là một loại sứ thủy tinh với độ bền cao nhưng vẫn duy trì tính thẩm mỹ tốt và tính tương hợp sinh học [2]. Hiện nay, lithium disilicate là một trong những vật liệu sứ được sử dụng phổ biến nhất trong nha khoa. Độ trong suốt cao của sứ thủy tinh lithium disilicate làm chúng trở thành sự lựa chọn tối ưu để phục hồi răng trước [3]. IPS e.max Press được giới thiệu bởi hãng Ivoclar Vivadent năm 2005, là một loại sứ thủy tinh lithium disilicate được ứng dụng rộng rãi và đạt tỉ lệ thành công $96 \%$ trong thực hành lâm sàng cho đến nay.

Gần đây, các nhà nghiên cứu đã phát triển dòng sứ lithium disilicate mới, trong đó, GC 
Initial Lisi Press với những cải tiến về cấu trúc vi thể giúp phân tán đều mật độ các tinh thể. Các thông số được đưa ra bởi nhà sản xuất cho thấy sự cải tiến về tính chất cơ học như độ bền uốn, độ kháng mài mòn... của vật liệu so với sứ thủy tinh lithium disilicate trước đây [4]. Tuy nhiên, vì là vật liệu mới, có rất ít nghiên cứu độc lập, đa số các dữ liệu về tính chất vật lý của chúng được công bố riêng bởi các nhà sản xuất.

Trong quy trình xử lý mặt dán sứ, xoi mòn bằng acid hydrofluoric (HF) là một quá trình động học làm thay đổi cấu trúc cơ học, bề mặt của sứ. Về cơ bản, HF phản ứng với pha thủy tinh, loại bỏ chọn lọc và làm bộc lộ cấu trúc tinh thể [5]. Tuy nhiên, việc xoi mòn quá mức có thể làm yếu sứ thủy tinh cũng như tạo các lỗ hổng trên bề mặt liên kết của phục hình răng sứ. Vì thế, nhiều nghiên cứu chứng minh rằng việc tạo bề mặt không đều như mong muốn cho sứ lithium disilicate đạt được bằng cách xoi mòn $\mathrm{HF}$ $5 \%$ trong 20 giầy [6]. Chúng tôi thực hiện nghiên cứu này với mục tiêu " Đánh giá độ cứng Vickers và cấu trúc bề mặt của 2 loại sứ thuỷ tinh lithium disilicate (GC Initial Lisi Press và IPS E.max Press) trước và sau khi xử lý HF 5\%".

\section{II. ĐỐI TƯợNG VÀ PHƯƠNG PHÁP NGHIÊN CỨU}

Nghiên cứu in vitro có nhóm chứng. Nghiên cứu được tiến hành trên các đĩa sứ thủy tinh chế tác bằng kỹ thuật ép nóng gồm hai nhóm: GC Initial Lisi Press (LP) và IPS e.max Press (EP).

\section{Quy trình chuẩn bị sứ}

Chuẩn bị các đĩa sứ: Thực hiên mẫu sáp tròn với kích thước $4 \times 2 \mathrm{~mm}$ và đặt vào khuôn. Dung dịch đúc được trộn và cho vào khuôn. Sau 30 phút, gõ bỏ khuôn, đưa vào máy (Programat EP3010) thực hiện quy trình nung để làm chảy sáp ở nhiệt độ $850^{\circ} \mathrm{C}$ trong 60 phút. Sau quá trình nung, đặt thỏi sứ tiền chế vào bên trong lõi và thực hiện quy trình ép nóng. Sau khi hoàn tất, làm nguội ở nhiệt độ phòng và lấy mẫu sứ từ bên trong lõi.

Đánh bóng bề mặt sứ bằng giấy nhám với độ mịn từ 400 đến 1000 grit dưới nguồn nước cất để loại bỏ các bất thường cũng như dấu trây xước, tạo ra bề mặt đồng nhất.

Quan sát dưới kính hiển vi điện tử quét (SEM). 12 đĩa sứ lithium disilicate kích thước $4 \times 2 \mathrm{~mm}$ từ mỗi loại sứ ép được chia làm hai nhóm có và không xử lý HF $5 \%(\mathrm{n}=3)$.

Với nhóm cần xử lý HF 5\%: Bề mặt sứ sau khi làm sạch được xoi mòn bằng cách bôi gel $\mathrm{HF}$ 5\% (IPS Etching Gel, Ivoclar Vivadent) trong 20 giây. Sau đó rửa dưới vòi nước chảy, loại bỏ hoàn toàn hơi nước bằng máy rửa siêu âm trong 15 phút. Quan sát SEM để đánh giá cấu trúc bề mặt sứ.

Thử nghiệm độ cứng Vickers. 40 đĩa sứ lithium disilicate từ mối loại sứ ép GC Initial Lisi Press (LP) và IPS e.max Press (EP) được chia làm hai nhóm trước và sau khi xử lý HF $5 \%$ $(n=10)$. Thử nghiệm độ cứng Vickers được dựa theo nghiên cứu của Alammari và cs (2018):

- Các đĩa sứ được đặt cố định trên một khối hình trụ.

- Sử dụng máy đo độ cứng Vickers (FM-700, Future-Tech Corp, Kanagawa, Nhật Bản) với lực tải 9,807N trong 10 giây tạo nên 5 vết lõm trển mỗi mẫu.

- Các vết lõm tạo ra được đo đường chéo thông qua phần mềm máy tính ở độ phóng đại 5000x.

- Giá trị trung bình độ cứng Vickers được tính cho từng mẫu.

Đo độ cứng Vickers được thực hiện bằng 1 mũi kim cương hình chóp 4 cạnh, góc giữa 2 mặt chóp đối diện là $136^{\circ}$. Đường chéo d1 và d2 được hiển thị và ghi lại trên máy tính. Từ đó tính được số trung bình giữa hai đường chéo. Độ cứng Vickers được tính dựa vào công thức: $\mathrm{HV}=$ $0,01891 \mathrm{~F} / \mathrm{d}^{2}$ (đđơn vị HV). Trong đó:F là lực được dùng để kiểm tra được tính bằng $\mathrm{N}$; $\mathrm{d}$ là chiều dài trung bình hai đường chéo của vết lõm được tính bằng đơn vị mm.

\section{KẾT QUẢ NGHIÊN CỨU \\ Độ cứng Vickers trước và sau xử lý HF $5 \%$ (Bảng 1)}

- Trước khi xử lý HF 5\%, độ cứng Vicker của 2 nhóm GC Initial Lisi Press LP và IPS e.max Press EP khác biệt không có ý nghĩa thống kê.

- Sau khi xử lý HF 5\%, độ cứng Vickers của cả 2 nhóm LP và EP đều giảm có ý nghĩa thống kê so với trước khi xử lý HF 5\%.

- Sau khi xử lý HF 5\% nhóm LP có độ cứng Vickers lớn hơn có ý nghĩa thống kê so với nhóm EP.

Bảng 1: Độ cứng Vickers

\begin{tabular}{|c|c|c|c|}
\hline \multirow{2}{*}{ Sứ } & \multicolumn{2}{|c|}{ Độ cứng Vickers (HV) } & Giá trị p \\
\cline { 2 - 4 } & $\begin{array}{c}\text { Trước xử lý } \\
\text { HF } 5 \%\end{array}$ & $\begin{array}{c}\text { Sau xứ lý HF } \\
5 \%\end{array}$ & \\
\hline $\begin{array}{c}\text { GC Initial } \\
\text { Lisi Press } \\
(n=10)\end{array}$ & $\begin{array}{c}637,82 \pm \\
5,28\end{array}$ & $628,04 \pm 7,16$ & 0,003 \\
\hline $\begin{array}{c}\text { IPS e.max } \\
\begin{array}{c}\text { Press } \\
(n=10)\end{array}\end{array}$ & $\begin{array}{c}627,92 \pm \\
14,47\end{array}$ & $\begin{array}{c}609,72 \pm \\
17,00\end{array}$ & 0,019 \\
\hline Giá trị p & 0,057 & 0,008 & \\
\hline
\end{tabular}

Phép kiểm t-test độc lập 


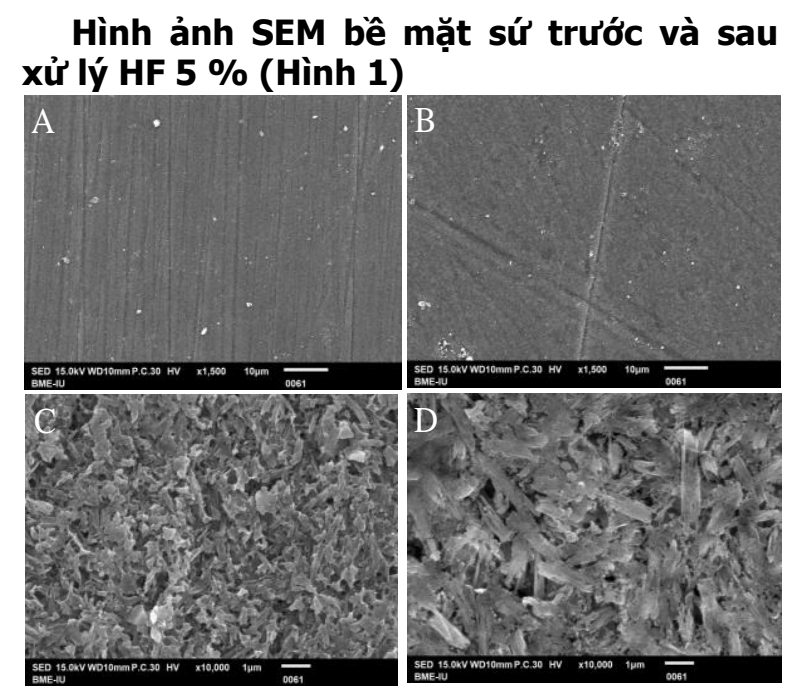

Hình 1: Hinh ảnh quan sát SEM.

Trước xử lý HF 5\% : A. LP, B. EP; Sau xử lý HF 5\%: C. LP, D.EP

Trước khi xử lý HF 5\%, nhóm LP (A) cho bề mặt mịn đều, rãnh cạn hơn so với nhóm EP (B).

Sau khi xử lý HF $5 \%$, nhóm LP (C) có bề mặt với kích thước các tinh thể nhỏ và đều so với nhóm EP (D).

\section{BÀN LUẬN}

Độ cứng là đặc tính cơ học điển hình ảnh hưởng lên sự thành công của vật liệu lâm sàng. Vật liệu có độ cứng bề mặt thấp hơn có thể dễ dàng bị hỏng và sự hỏng bề mặt gây ra độ mỏi, làm giảm tỉ lệ thành công [7]. Độ cứng là thước đo khả năng chống biến dạng dẻo và được đo bằng lực trên một đơn vị diện tích [8].

Trước khi xử lý HF 5\%, độ cứng Vickers của hai nhóm sứ LP và EP không có sự khác biệt có ý nghĩa thống kê. Kết quả quan sát SEM cho thây nhóm LP có bề mặt mịn đêu, tạo các đường sọc cạn do đánh bóng và nhóm EP tạo thành các đường sọc lớn hơn. Mặc dù cấu trúc bề mặt của EP xuất hiện những vết rãnh lớn hơn LP, nhưng cả 2 nhóm đều không tạo các cấu trúc lổ rỗng lớn. Điều này có thể lý giải là do hiệu ứng đông đặc của sứ thủy tinh ở dạng mềm được ép ở nhiệt độ cao dưới chân không hay mật độ phân tử khác nhau nên bọt khí có thể lọt vào mấu sứ ở giai đoạn ép nhiệt khác nhau. Việc xuất hiện cấu trúc không đều, vết rãnh nhỏ ở giai đoạn trước khi xử lý HF $5 \%$ không làm ảnh hưởng nhiều đến độ cứng của hai nhóm LP và EP.

Sau khi xử lý với axit HF 5\%, độ cứng bề mặt của cả 2 loại sứ đều giảm có ý nghĩa thống kề. Việc xử lý axit HF làm hoà tan các pha thuỷ tinh, bộc lộ pha tinh thể lithium dislicate [5]. Kết quả quan sát SEM cho thây độ nhám, gồ ghề của bề mặt sứ tăng sau khi xử lý axit HF 5\% với các cấu trúc lỗ rỗng giữa các tinh thể có kích thước khác nhau. Sự phát triển của kích thước tinh thể trong quá trình thể hoá, và hình thành cấu trúc rỗng (độ xốp) ảnh hưởng bất lợi đến độ cứng sứ bằng cách hoạt động như một chất tập trung ứng suất. Như vậy việc xử lý bề mặt sứ với axit HF $5 \%$ nhằm tăng độ bám dính của sứ vào xi măng resin trong ứng dụng lâm sàng, những đã ảnh hưởng đến độ cứng của sứ. Kết quả nghiên cứu này tương đồng với nghiên cứu của Lubica và cs, (2019), nhóm tác giả này cho rằng cả hai loại sứ LP và EP đều có độ cứng bề măt giảm sau xữ lý với axit HF [9]. Như vậy, việc kiểm soát độ rỗng nên được xem xét khi chế tạo hay xử lý các vật liệu phục hình răng bằng sứ thủy tinh, để giảm tần suất gãy của sứ khi nhai thức ăn cứng.

Sau khi xử lý HF $5 \%$, các tinh thể lithium disilicate của nhóm EP định hướng ngẫu nhiên, không đều với nhiều cấu trúc lỗ rỗng khác nhau. Trong khi, nhóm LP có các tinh thể được sắp xếp đồng đều. Các tinh thể của LP có kích thước nhỏ hơn, đều và mật độ dày đặc hơn so với nhóm EP. Sự hình thành các tinh thể được xếp đồng bộ sẽ làm cho các vết nứt lan truyền theo hướng các phân tử dẫn đến tạo rãnh nứt quanh co hơn, vì thế cải thiện độ cứng. Mặt khác, khi các phân tử có khoảng cách xa nhau, đường nứt sẽ dễ dàng xuất hiện xen giữa các khoảng trống của các tinh thể.

Một thực tế là các tinh thể không chỉ thẳng hàng dọc theo hướng ép mà còn song song với bề mặt của các mẩu sứ được ép. Trong các trường hợp phục hồi răng bằng vật liệu sứ ép, cấu trúc các tinh thể được xếp đồng bộ, ít có khoảng trống giữa các tinh thể có khả năng tạo liên kết tốt hơn. Do đó, có thể cho rằng với loại kết cấu này sẽ gia tăng khả năng chống lại lực dọc. Điều này có thể giải thích độ cứng của sứ LP sau xử lý HF cao hơn sứ EP.

\section{KẾT LUẬN}

Kết quả nghiên cứu của chúng tôi cho thây cấu trúc vi thể bề mặt của sứ thuỷ tính lithium disilicate trước và sau khi xử lý HF $5 \%$ có sự tương quan đến sự khác biệt về độ cứng Vickers. Việc xứ lý HF \% làm tăng cấu trúc lố rỗng bề mặt và làm giảm độ cứng bề mặt của sứ.

\section{TÀI LIỆU THAM KHẢO}

1. Nikzad S, Azari A, Dehgan S. (2010). "Ceramic (Feldspathic \& IPS Empress II) vs. laboratory composite (Gradia) veneers; a comparison between their shear bond strength to enamel; an 
in vitro study". Journal of oral rehabilitation. $\mathbf{3 7}$ (7), pp 569-574.

2. Xiaoping $L$, Dongfeng $R$, Silikas N . (2014). "Effect of etching time and resin bond on the flexural strength of IPS e.max Press glass ceramic". Dental Materials. 30 (12), pp. e330-e336.

3. Kurian BP, Johns M, Hareesh MT. (2018). "Lithium Disilicate Ceramic - An Overview". The Journal of Prosthetic and Implant Dentistry. 1 (2)

4. Carrabba M. et al. (2017). "Flexural strength of CAD/CAM and pressed novel lithium disilicate". Journal of Oral and Craniofacial Biomaterials Sciences. 33, pp. e16.

5. Prochnow C, Venturini A B, Grasel R, Gundel A, Bottino M C, Valandro L F. (2018), "Adhesion to a Lithium Disilicate Glass Ceramic Etched with Hydrofluoric Acid at Distinct
Concentrations". Brazilian Dental Journal. 29 (5) pp. 492-499.

6. Kalavacharla V K, Lawson N C, Ramp L C, Burgess J O. (2015), "Influence of Etching Protocol and Silane Treatment with a Universal Adhesive on Lithium Disilicate Bond Strength". Oper Dent. 40 (4), pp. 372-378.

7.Sakaguchi R. et al. (2011). Craig's Restorative Dental Materials, 13th Edition -Mosby (2011), Vol. 11.

8. Rawls KJ: Mechanical properties of dental materials In Anusavice KJ (ed): Phillips' Science of Dental Materials (ed 11). St. Louis, W.B. Saunders, 2003, pp. 69-143

9. Hallmann $L$, Ulmer $P$, Gerngross MD, Jetter J, Mintrone M, Lehmann F, Kern M. Properties of hot-pressed lithium silicate glass-ceramics. (2019). Dental materials. 35, 713-729

\section{THỰC TRANG TIỀN TĂNG HUYẾT ÁP, TĂNG HUYÊT ÁP Ở NGƯờII DÂN TỬ 30 TUỔI TRỞ LÊN TẠI QUÂ̂N HẢI CHÂU, THÀNH PHỐ ĐÀ NÃ̃NG}

\section{TÓM TẮT}

Mục tiêu xác định tỷ lệ tiền tăng huyết áp, tăng huyết áp của người dân từ 30 tuổi trở lên tai Quấn Hải Châu, thành phố Đà Nẵng. Đối tượng và phương pháp: Nghiên cứu mô tả cắt ngang trên 495 đối tượng là người dân từ 30 tuổi trở lên. Kết quả: Tỷ lệ tiền tăng huyết áp ở đối tượng nghiên cứu là $19 \%$ và̀ tỷ lê tăng huyết áp là $21 \%$. Kết luân: Tỷ lệ tiền tăng huyết áp và tỷ lệ tăng huyết áp gần tương đương nhau do đó cần tiến hành các biện pháp can thiệp để giải quyết vấn đề này kịp thời. Nẵng.

Tư khóa: tiền tăng huyết áp, tăng huyết áp, Đà

\section{SUMMARY}

\section{THE SITUATION OF PRECAUTIONS OF PRECAUTIONS, RISK PRESSURE IN PEOPLE AGE 30 AND OVER IN HAI CHAU DISTRICT, DA NANG CITY}

Objective: to determine the rate of prehypertension and hypertension of people aged 30 years and older in Hai Chau District, Da Nang city. Subjects and methods: A cross-sectional descriptive study on 495 subjects who were people aged 30 years and older. Results: The rate of prehypertension in the study subjects was $19 \%$ and the rate of hypertension was $21 \%$. Conclusion: Prehypertension and hypertension rate are almost similar,

${ }^{1}$ Trường Đại học Kỹ thuật Y - Dược Đà Nẵng Chiu trách nhiệm chính: Nguyễn Khắc Minh Email: minh@dhktyduocdn.edu.vn Ngày nhận bài: 10.5.2021

Ngày phản biện khoa học: 28.6.2021

Ngày duyệt bài: 8.7.2021
Trần Anh Quốc ${ }^{1}$, Nguyễn Văn Song1, Trần Đình Trung ${ }^{1}$, Nguyễn Khắc Minh ${ }^{1}$

so it is necessary to conduct interventions to solve this problem promptly.

Keywords: pre-hypertension, hypertension, Da Nang.

\section{I. ĐĂT VẤN ĐỀ}

Tăng huyết áp đang trở thành một vấn đề thời sự và phổ biến ở cộng đồng hiện nay, là nguyên nhân gây tử vong và tàn phế, để lại hậu quả nă̆ng nề về tinh thần và kinh tế cho gia đình và xã hội [1]. Trên thế giới tỷ lệ tăng huyết áp chiếm khoảng $8-18 \%$ dân số và có sự khác nhau ở các quốc gia. Theo Tổ chức $Y$ tế thế giới, mỗi năm có khoảng 9 triêu người chết do tăng huyết áp, chiếm $12,8 \%$ tử vong toàn cầu, tăng huyết áp đã và đang trở thành nguy cơ hàng đầu của các biến chứng tim mạch[9]. Chúng tôi tiến hành nghiên cứu này với mục tiêu: xác định tỷ lê tiền tăng huyêt áp, tăng huyêt áp của người dân từ 30 tuôi trở lên tại Quận Hải Châu, thành phố Đà Nẵng.

\section{II. ĐỐI TƯợNG VÀ PHƯƠ'NG PHÁP NGHIÊN CỨU}

\section{1. Đối tượng nghiên cứu}

Tiêu chuẩn lựa chọn: Người dân từ 30 tuổi trở lên thuộc các phường của Quận Hải Châu, Tp. Đà Nẵng, tự nguyện tham gia nghiên cứu.

Tiêu chuân loai trừ

- Đối tượng nghiên cứu từ chối tham gia.

- Người khiếm thính.

- Đang mắc bệnh nặng không thể tham gia hoặc không sinh sổng thường xuyên tại khu vực.

- Không có khả năng tham gia vào nghiên cứu.

2.2. Địa điểm nghiên cứu: trạm y tế ở 5 